\title{
Research on Prediction Model of Conical Pick Cutting Force Based on Coulomb-Mohr Criterion
}

\author{
S.J. Wang ${ }^{1, *}$, X.M. Zong ${ }^{1,2}$, B. He ${ }^{1,2}$ and K.X. Kang ${ }^{1}$ \\ ${ }^{1}$ Jiangsu Xugong Construction Machinery Research Institute Co., Ltd, Xuzhou 221004, China \\ ${ }^{2}$ Sate Key Laboratory of Intelligent Manufacturing of Advanced Construction Machinery, Xuzhou 221004, China
}

\begin{abstract}
Cutting force is one of the most important influence factors that affect the efficiency and service life of the conical pick. In order to accurately calculate the cutting force, through theoretical analysis and experimental research, a cutting force model consisted of the basic fracture parameters of rock, geometric parameters and installation parameters of pick is proposed based on the coulomb-mohr criterion, and the calculation model is corrected by whole cutting experimental data, and validated the corrected model by orthogonal test, the result show that the error of the cutting force model is about $10 \%$, which meets the requirements of engineering data. At the same time, significant analysis on the cutting force is obtained by range method, cutting depth $h>$ semi-tip angle $\beta>$ cutting angle $\alpha$.
\end{abstract}

\section{Introduction}

As a crushing tool of brittle materials such as coal and rock, conical pick is a key component affecting the working performance of mining machinery such as coal shearer, roadheader and trench cutter ${ }^{[1-2]}$, the research on cutting force of pick has a certain theoretical guiding significance for customized design of pick, arrangement design of rotor installation and matching design of cutting torque and propulsion force of the whole machine under different working conditions.

At present, domestic and foreign scholars mainly study the cutting force of pick under the condition of straight line and equal depth of cutting. The corresponding cutting force models are derived based on the tensile failure model, which can better reflect the breaking process of coal and rock, but the calculation results accuracy is poor. Evans ${ }^{[3]}$ took the lead in putting forward a pick cutting force model based on rock tensile failure theory, but the cutting angle of the pick is 0 degree, the testing situation is quite different from the actual working condition. GÖktan R.M. ${ }^{[4-5]}$ modified Evans model, and proposed a semi-empirical cutting theoretical model considering friction between pick and rock. Based on fracture mechanics, R.H. Bao et al. $^{[6]}$ proposed a mechanical model for estimating the cutting force, and it is considered that the maximum cutting force has a power law relationship with the depth of cut. Based on cutting test data and principal component regression analysis method, domestic scholars Liang Yun-pei et al. ${ }^{[7-8]}$ studied the factors such as depth of cut, cutting distance and cutting angle, which influences on the cutting force. Wang Li-ping et al. ${ }^{[9-10]}$ established the calculation model of cutting force under symmetrical or asymmetrical condition of plane cutting slot based on Evans model.
Based on the load spectrum of single-pick cutting force test, Liu Chun-sheng et al. ${ }^{[11]}$ adopted the load superposition algorithm combining experiment with theory, the cutting torque of the whole machine is calculated, which provided a train of thought for the power design of the whole machine.

On the basis of Coulomb-Mohr failure criterion of rock, the author has established a cutting force calculation model with rock fracture parameters, pick geometry parameters and installation parameters as variables by combining theoretical analysis and experimental research.

\subsection{Analysis of pick motion characteristics}

The depth of cut hi changes with time is the characteristic of rotary cutting, this paper uses the plane rotation cutting scheme to simplify, it meets the changing requirement of depth of cut hi, as shown in fig. 1., with $\mathrm{O}$ point as the rotary center, the conditions for cutting pick to penetrate into rock are as follows.

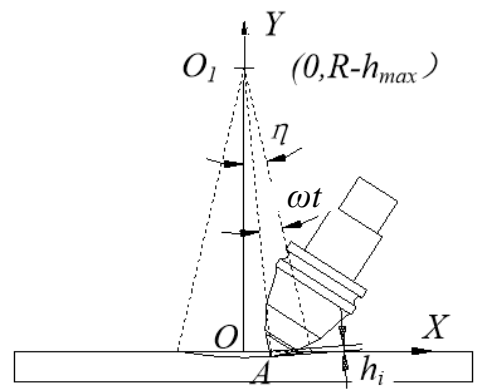

Fig.1. Face cutting test

$$
\eta=\cos ^{-1}\left(\frac{R-h_{\max }}{R}\right)
$$

$\mathrm{R}$ - the cutting radius of the pick, $\mathrm{mm}$.

$\mathrm{H}_{\max }$ - the maximum depth of cut, $\mathrm{mm}$. 
On this basis, the formulas for calculating depth of cut at different times are established.

$$
h_{i}=R-\frac{R-h_{\max }}{\cos (\eta-\omega t)}
$$

In the cutting process of the pick, the area in front of the two side edges of the alloy head which contacts the rock directly, and projects in normal and tangential directions respectively, as shown in Fig. 2b. and Fig. 2c.

a)

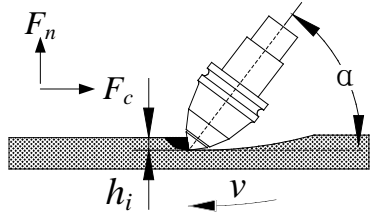

b)

c)

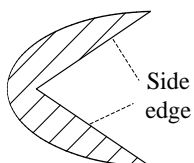

Side

Side edge

Fig.2. Contact area:a) Force diagram of pick cutting process b) invasion indentation of pick c) tangential shear zone

The cutting force for crushing rock includes: $F_{n}$ - Normal force, $F_{c}$ - Tangential force.

\subsubsection{Establishment Normal force model of pick}

Normal force is not only related to rock mechanical properties, but also related to rock indentation area and motion parameters, as shown in Fig. 4a, and the rock indentation area is decided by the parameters such as half cone angle of alloy head $\varphi$, diameter of alloy head d, cutting angle $\alpha$ and depth of cut h, etc ${ }^{[9]}$.

a)

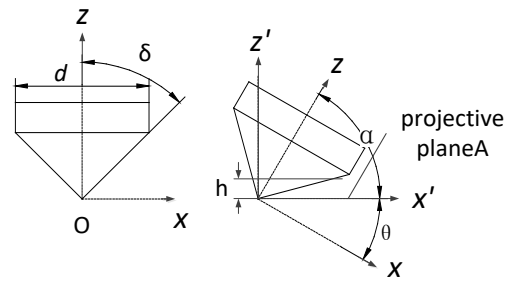

b)

B

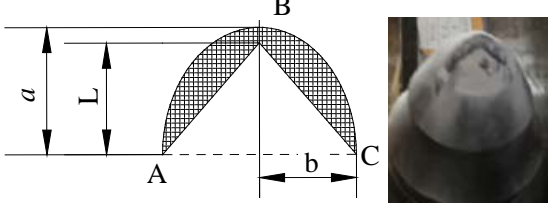

Fig.3. Intrusion area of pick: a) Schematic diagram of pick intrusing into rock b) Contrast map of invasive area of pick As shown in Fig. 4a., the pick intrudes into the rock at a certain cutting angle $\alpha$, the effective indentation of rock is shown in Fig. 3b., effective indentation is the projection of the contact area between the pick alloy head and rock in the plane perpendicular to the direction of the pick invasion (projection plane A), A closed region consisting of half elliptic arc $\mathrm{ABC}$, segment $\mathrm{AB}$ and $\mathrm{BC}$.

According to the formula of coordinate rotation, the coordinates of the alloy head after rotation angle are calculated, the equation of inclined cone is obtained by substituting the equation of cone. $\left(k^{2} \cos ^{2} \theta-\sin ^{2} \theta\right) x^{2}-2\left(k^{2}+1\right) \sin \theta \cos \theta x z+k^{2} y^{2}=\left(\cos ^{2} \theta-k^{2} \sin ^{2} \theta\right) z^{2}$

$\mathrm{k}$-slope of cutter alloy head cone, $\mathrm{k}=\tan \delta$.

$\theta$-the rotation angle of Pick, $\theta=90-\alpha,{ }^{\circ}$.

As shown in Fig. 4b., When the depth of cut of the pick is $\mathrm{h}$, formula (3) is simplified to obtain that the indentation region of rock satisfies elliptic equation.

$$
\begin{gathered}
\frac{(x-\mathrm{L})^{2}}{a^{2}}+\frac{y^{2}}{b^{2}}=1 \\
\left\{\begin{array}{l}
a=\frac{k h}{k^{2} \cos ^{2} \theta-\sin ^{2} \theta} \\
b=\frac{h}{\sqrt{k^{2} \cos ^{2} \theta-\sin ^{2} \theta}} \\
\mathrm{L}=\frac{\left(k^{2}+1\right) \sin \theta \cos \theta}{k^{2} \cos ^{2} \theta-\sin ^{2} \theta} h
\end{array}\right.
\end{gathered}
$$

According to the rock hardness measurement formula and the deduction formula of the invasive area during the cutting process of the pick, the mathematical model of the invasive force of the pick is obtained.

$$
F_{n}=\sigma_{n} S_{n}=\sigma_{n}\left(\frac{\pi a b}{2}-L b\right)
$$

Where

$a$ - long axis of ellipse, $\mathrm{mm}$.

$b$ - short axis of ellipse, mm.

$\mathrm{L}$ - distance from tip to ellipse center line, $\mathrm{mm}$.

$\sigma_{n}$-rock hardness, which needs to be measured, MPa. $\mathrm{mm}^{2}$.

$S_{n}$ - projection of contact area between pick and rock,

Rock hardness is the ability to resist the pressure of the pick intruding into its interior, which represents the stress of the rock when it is subjected to local failure, the compressive strength is the stress of failure of the whole rock surface, which is greatly affected by rock defects, because the rock cut by pick is partially broken, the rock's compressive hardness is more practical than the uniaxial compressive strength [12].

\subsubsection{Establishment Tangential force model of pick}

The Tangential force is partly the friction between the pick and the rock, and partly the thrust of the pick when the rock is sheared.

$$
F_{c}=F_{c}^{\prime}+F_{c}^{\prime \prime}
$$

$\mathrm{F}_{\mathrm{C}}{ }^{\prime}$ - Friction is between pick and rock.

$\mathrm{F}_{\mathrm{C}}{ }^{\prime}{ }^{\prime}$ - Thrust of the pick when the rock is sheared.

$$
F_{c}^{\prime}=F_{n} f=\sigma_{n}\left(\frac{\pi a b}{2}-L b\right) f
$$

Rock shear failure follows Coulomb-Moore failure criterion, as shown in Fig. 5., it is assumed that the cutting process satisfies the following conditions: the internal composition of the rock is uniform, the contact stress q between pick and rock is evenly distributed, and the shear fracture surface of rock follows the Coulomb- 
Mohr criterion. the fracture surface starts from the tip of the pick and develops upward to the free surface at an angle of $\theta_{f}$ with the cutting surface, assuming that the blade is sharp and only the surface before the two side edges is contacted with the rock ${ }^{[13]}$.

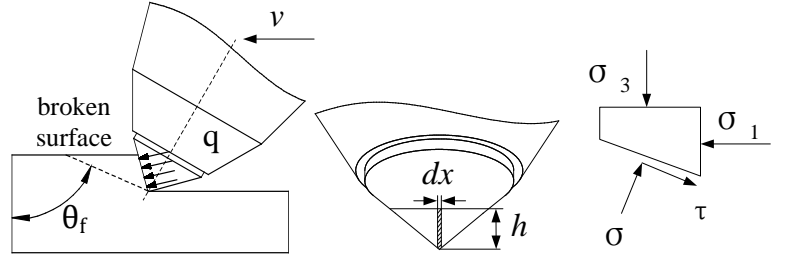

$\sigma_{1}$ - principal stress in cutting direction of rock. $\sigma_{3}$ - stress on Free Surface of Rock. $\theta_{f}$ - breaking angle.

$\sigma$ - Principal stress of failure surface. $\tau$ - Shear force on failure surface.

Fig.4. Analysis of rock shear failure

Since the free surface stress of rock is zero, the normal and shear stress of the failure surface are calculated according to the Mohr stress circle ${ }^{[14]}$.

$$
\left\{\begin{array}{l}
\sigma=\frac{\sigma_{1}}{2}+\frac{\sigma_{1}}{2} \cos 2 \theta_{f} \\
\tau=\frac{\sigma_{1}}{2} \sin 2 \theta_{f} \\
\theta_{f}=45+\frac{\varphi}{2}
\end{array}\right.
$$

To obtain the limit shear stress of the rock, the determined cohesive force and friction angle of the rock are substituted into the Coulomb shear strength formula.

$$
\tau_{f}=c+\sigma \tan \varphi
$$

According to the Mohr-Coulomb failure criterion of rocks, rock is in the limit equilibrium when $\tau=\tau_{f}$ happens on the failure surface, The critical normal $\sigma_{c}$ for shear failure of rock is obtained.

$$
\sigma_{c}=\frac{2 c}{\sin (90+\varphi)-\tan \varphi(1+\cos (90+\varphi))}
$$

As shown in Fig. 5., according to the different depths of the intrusion into the rock, the shape of the contact area between the alloy head and the rock is different during the cutting process.

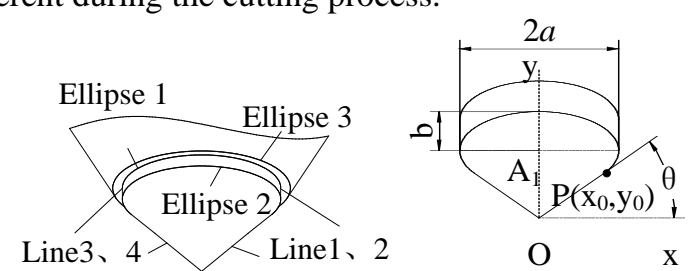

Fig.5. Crusher area of rock shearing failure

When the cutting thickness $h<y_{0}$, the cutting groove is triangular and the contact area $S_{c}$ formula is as follows.

$$
S_{c}=h^{2} \tan (90-\theta)
$$

When the cutting thickness $y_{0} \leq h<h_{2}$, the cutting groove is a triangle and a trapezoid, and the contact area $S_{c}$ is calculated as follows.

$$
S_{c}=y_{0}^{2} \tan (90-\theta)+\left(x_{0}+x_{1}\right)\left(h-y_{0}\right)
$$

Where

$$
\begin{aligned}
& \theta=\tan ^{-1}\left(\frac{\sqrt{4\left(l_{2} \sin \alpha\right)^{2}-\left(d_{2} \cos \alpha\right)^{2}}}{d_{2}}\right) \\
& h_{2}=\frac{\cos ^{2} \alpha\left(d_{3}{ }^{2}-d_{2}{ }^{2}\right)+4 \sin ^{2} \alpha\left(l_{3}{ }^{2}-l_{2}^{2}\right)}{8 \sin \left(l_{3}-l_{2}\right)}
\end{aligned}
$$

$l_{2}$ - length of cone, $\mathrm{mm}$.

$l_{3}$ - length of alloy head, $\mathrm{mm}$.

$d_{2}$ - Maximum diameter of cone, $\mathrm{mm}$.

$d_{3}$ - Minimum diameter of pick body, mm.

$h_{2}$-Maximum depth of pick that do not participate in cutting progress, $\mathrm{mm}$.

Based on the above cutting area and critical normal stress, the calculation model of cutting tangential cutting force is obtained.

$$
F_{c}^{\prime \prime}=\left\{\begin{array}{l}
\sigma_{c} h^{2} \tan (90-\theta) \text { when } h \leq y_{0} \\
\sigma_{c}\left[y_{0}{ }^{2} \tan (90-\theta)+\left(x_{0}+x_{1}\right)\left(h-y_{0}\right)\right] \text { when } y_{0}<h \leq h_{2}
\end{array}\right.
$$

According to the model, there is a positive proportional quadratic relationship between thrust of rock $F_{c}{ }^{u}$ and cutting thickness $h$, and there are complex trigonometric relationships between thrust of rock $F_{c}{ }^{n}$ and the semi-cone angle of the alloy head $\beta$, cutting angle $\alpha$, which are consistent with the results of reference ${ }^{[15]}$.

In summary, the calculation model of tangential cutting force of pick is as follows.

$$
F_{c}=\left\{\begin{array}{l}
\sigma_{c} h^{2} \tan (90-\theta)+\sigma_{n} f\left(\frac{\pi a b}{2}-\mathrm{L} b\right) \text { when } h \leq y_{0} \\
\sigma_{c}\left[y_{0}{ }^{2} \tan (90-\theta)+\left(x_{0}+x_{1}\right)\left(h-y_{0}\right)\right]+\sigma_{n} f\left(\frac{\pi a b}{2}-L b\right) \text { when } y_{0}<h \leq h_{2}
\end{array}\right.
$$

Table1 Parameters of static pressure

\begin{tabular}{|c|c|c|c|c|}
\hline \multicolumn{3}{|c|}{ Pick specification } & \multicolumn{2}{c|}{ Test machine parameters } \\
\hline $\boldsymbol{\beta}$ & $\begin{array}{c}\text { diamete } \\
\mathbf{r} / \mathbf{m m}\end{array}$ & $\begin{array}{c}\boldsymbol{\alpha} \\
/^{\circ}\end{array}$ & $\begin{array}{c}\text { velocity } \\
/ \mathbf{s}\end{array}$ & $\begin{array}{c}\text { displacement } \\
/ \mathbf{m m}\end{array}$ \\
\hline $45^{\circ}$ & 22 & 53 & 1 & 5 \\
\hline
\end{tabular}

According to the definition of rock hardness and test data, the rock hardness is calculated.

$$
\sigma_{n}=\frac{F_{n}}{S_{n}}
$$

\begin{tabular}{|c|c|c|c|c|}
\hline $\begin{array}{c}\text { Serial } \\
\text { number }\end{array}$ & $\begin{array}{c}\text { Area } S_{n} \\
\left(\mathrm{~mm}^{2}\right)\end{array}$ & $\begin{array}{c}F_{n} \\
(\mathrm{KN})\end{array}$ & $\sigma_{n}$ (Mpa) & $\begin{array}{c}\overline{\sigma_{n}} \\
(\mathbf{M P a})\end{array}$ \\
\hline 1 & 21.7 & 3.8 & 176.2 & \multirow{3}{*}{179.8} \\
\hline 2 & 86.8 & 15.2 & 174.8 & \\
\hline 3 & 148.6 & 28.0 & 188.4 & \\
\hline
\end{tabular}

Where

$F_{n}$ - Pressure, $\mathrm{N}$.

$S_{n}$ - Projection of the pick invading rock area on the free plane of rock, $\mathrm{mm}^{2}$.

As shown in Table 2, the results are slightly dispersed, but the mean hardness of rock at different cutting angles is consistent.

Table2 Results of rock static pressure 
Through triaxial compression tests under different confining pressures, mohr circle envelopes of rocks under different confining pressures can be obtained, as shown in Fig. 8. By calculating the envelope equation of Mohr circle, the cohesion and internal friction angle of rock are obtained. The calculation results are shown in Table 3.

Table3 Results of red sandstone shearing test

\begin{tabular}{|c|c|c|c|c|}
\hline $\boldsymbol{\sigma}_{3}$ (Mpa) & $\boldsymbol{\sigma}_{\mathbf{1}}$ (Mpa) & $\boldsymbol{c}(\mathbf{M P a})$ & $\boldsymbol{\varphi}\left({ }^{\circ}\right)$ & $\boldsymbol{\sigma}_{\boldsymbol{c}}(\mathbf{M p a})$ \\
\hline 5 & 112.8 & & & \\
\hline 10 & 133.8 & \multirow{2}{*}{28.3} & \multirow{2}{*}{32} & 102.1 \\
\hline 20 & 173.12 & & & \\
\hline 30 & 198.22 & & & \\
\hline
\end{tabular}

\subsection{Verification of Cutting Model}

Because the model of cutting force and cutting force is quasi-static test result, there is a certain gap between the cutting speed and the actual cutting process, so the cutting force model is modified by using the experimental data.

\subsubsection{Brief description of test scheme}

According to the theoretical analysis model, the corresponding rotary cutting test-bed is built, as shown in Fig. 6.

Test scheme: Design different angle tooling to achieve different cutting angles of the pick, choose three kinds of pick with different half cone angle of alloy head, the hydraulic cylinder is used to adjust the cutting thickness of the pick, use pump and motor to adjust cutting speed. During the test, the distance between the center line of the adjacent cutting slot is more than 40 $\mathrm{mm}$, in this way, the adjacent cutting slots are avoided to penetrate each other and affect the cutting force, the cutting force is collected by three-way force sensor, and the data acquisition frequency is $256 \mathrm{HZ}$, Meanwhile, the radial and tangential cutting forces are collected during the cutting process.

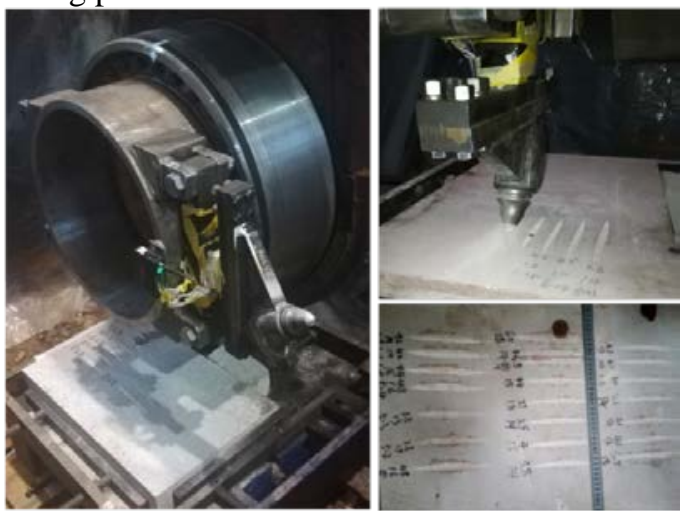

Fig.6. Test bench of rotary cutting

\subsubsection{Calculation of Velocity Compensation Coefficient}

Relevant parameters of the test: cutting angle $\alpha=63^{\circ}$, semi-cone angle of alloy head $\beta=45^{\circ}$, alloy head diameterd $=22 \mathrm{~mm}$, The actual speed of the whole machine is $15 \sim 16 \mathrm{r} / \mathrm{min}$, Maximum cutting thickness is $4.2 \mathrm{~mm}$, cutting medium is red sandstone. In order to eliminate the influence of rock inhomogeneity on test results, the mean cutting force of the same cutting thickness is selected and compared with the theoretical results after polynomial fitting, Calculation of velocity compensation coefficient $k_{c}$ and $k_{n}$, The calculation results are shown in Table 4 .

Table4 Comparison and correction of cutting force

\begin{tabular}{|c|c|c|c|c|c|c|c|c|c|}
\hline \multirow{2}{*}{$t / s$} & \multirow{2}{*}{$h_{i} / \mathbf{m m}$} & \multirow{2}{*}{$S_{c} / \mathrm{mm}^{2}$} & \multirow{2}{*}{$S_{n} / \mathbf{m m}^{2}$} & \multicolumn{2}{|c|}{ Theoretical calculation } & \multicolumn{2}{|c|}{ experimental data } & \multirow{2}{*}{$\boldsymbol{k}_{c}$} & \multirow{2}{*}{$k_{n}$} \\
\hline & & & & $F_{c} / \mathbf{N}$ & $F_{n} / \mathbf{N}$ & $F_{c} / \mathbf{N}$ & $F_{n} / \mathbf{N}$ & & \\
\hline 0.34 & 0 & 0 & 0 & 0 & 0 & 0 & 0 & - & - \\
\hline 0.35 & 0.2 & 0.05 & 0.07 & 8.3 & 12.0 & 44.8 & 73.2 & - & - \\
\hline 0.36 & 1.8 & 4.23 & 5.48 & 673.8 & 969.4 & 787.6 & 1060.3 & 1.17 & 1.09 \\
\hline 0.38 & 3.0 & 11.74 & 15.21 & 1871.8 & 2692.9 & 2031.9 & 3508.3 & 1.09 & 1.30 \\
\hline 0.39 & 3.7 & 17.86 & 23.14 & 2847.2 & 4096.2 & 3008.1 & 4924.7 & 1.06 & 1.20 \\
\hline 0.41 & 3.9 & 19.84 & 25.71 & 3163.3 & 4551.0 & 3049.9 & 4944.1 & 0.96 & 1.09 \\
\hline 0.42 & 3.7 & 17.86 & 23.14 & 2847.2 & 4096.2 & 2690.8 & 4301.4 & 0.95 & 1.05 \\
\hline 0.43 & 3.0 & 11.74 & 15.21 & 1871.8 & 2692.9 & 2064.6 & 3230.2 & 1.10 & 1.20 \\
\hline 0.45 & 1.8 & 4.23 & 5.48 & 673.8 & 969.4 & 804.7 & 1036.9 & 1.19 & 1.07 \\
\hline 0.46 & 0.2 & 0.05 & 0.07 & 8.3 & 12.0 & 46.2 & 65.9 & - & - \\
\hline 0.48 & 0 & 0 & 0 & 0 & 0 & 0 & 0 & - & - \\
\hline & & & & ralue & & & & 1.07 & 1.14 \\
\hline
\end{tabular}

As can be seen from the above table, influenced by the assumption that the pick tip is absolute cusp, When the cutting thickness $(\mathrm{h}=0.2 \mathrm{~mm})$ of the pick is small, the prediction model of cutting force has great error. When the cutting thickness is greater than $1.8 \mathrm{~mm}$, accuracy of cutting force model increases gradually. through the correction of several groups of test data, a relatively stable tangential cutting force correction coefficient $k_{c}=1.07$ and a normal intrusion force correction coefficient $k_{n}=1.14$ are obtained.

Cutting Thickness Obtained from Cutting Motion Equation, the modified cutting and intrusion resistance calculation model is substituted, the normal intrusive force and tangential cutting force during the operation are obtained and compared with the force curve during the actual cutting process. The results are shown in Fig. 7. 
a)

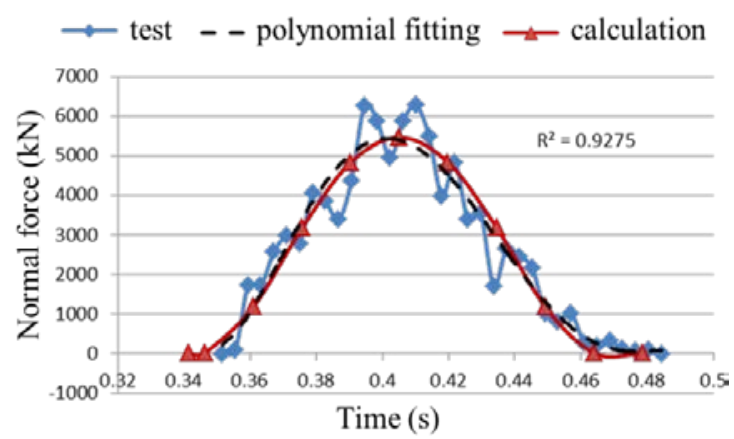

b)

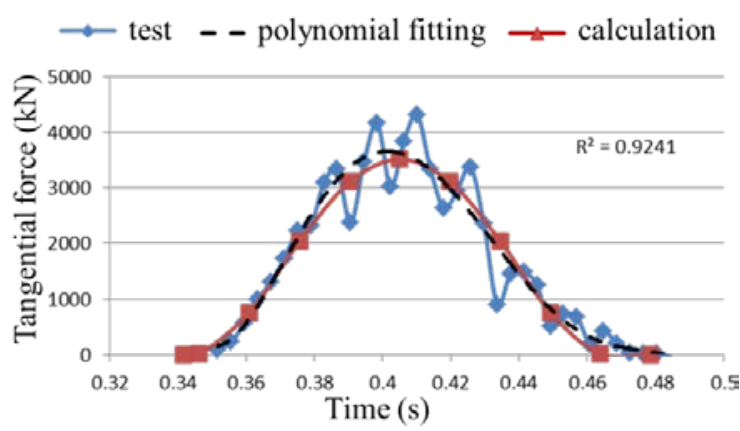

As can be seen from the figure above: The theoretical calculation is in good agreement with the fitting curve of the test data after the modification of the cutting process and the cutting process, it objectively reflects the quantitative re, the difference is the load fluctuation caused by "step" failure of rock ationship between the resistance of the cutting teeth and the cutting thickness during the cutting process.

\subsection{Contrastive Verification of Orthogonal Experiments}

Orthogonal test method was used, DOE test design with three factors and three levels was carried out for different cutting thickness h, half cone angle beta and cutting angle alpha of alloy head. The test scheme and results are shown in Table 5. The normal intrusive force and tangential cutting force are extracted when the maximum cutting thickness is extracted, and the maximum values of normal intrusive force and tangential cutting force are determined by polynomial fitting method. This paper mainly analyses the relationship between each factor and index, and the primary and secondary relationship of each factor.

Fig.7. Cutting force comparison: a) Normal Forces b) Cutting force

Table5 Plan and results of experiment

\begin{tabular}{|c|c|c|c|c|c|c|c|c|}
\hline \multirow{2}{*}{$\beta /^{\circ}$} & \multirow{2}{*}{$\alpha /^{\circ}$} & \multirow{2}{*}{$\mathbf{h} / \mathbf{m m}$} & \multicolumn{2}{|c|}{ Theoretical calculation } & \multicolumn{2}{|c|}{ experimental data } & \multicolumn{2}{|c|}{ Relative deviation $/ \%$} \\
\hline & & & $F_{n} / \mathbf{k N}$ & $\boldsymbol{F}_{c} / \mathbf{k N}$ & $F_{n} / \mathbf{k N}$ & $F_{c} / \mathbf{k N}$ & $F_{n}$ & $\boldsymbol{F}_{c}$ \\
\hline 35 & 53 & 2 & 753.3 & 678.1 & 694.3 & 636.8 & 8.5 & 6.5 \\
\hline 35 & 58 & 4 & 2396.8 & 2485.3 & 2192.9 & 2191.6 & 9.3 & 13.4 \\
\hline 35 & 63 & 6 & 4559.2 & 4848.6 & 4383.9 & 4481.1 & 4 & 8.2 \\
\hline 40 & 53 & 4 & 6078.6 & 4061.0 & 5707.6 & 3897.3 & 6.5 & 4.2 \\
\hline 40 & 58 & 6 & 8277.4 & 6285.9 & 8938.9 & 6961.1 & 7.4 & 9.7 \\
\hline 40 & 63 & 2 & 900.4 & 752.0 & 814.9 & 673.2 & 10.5 & 11.7 \\
\hline 45 & 53 & 6 & 28271.2 & 14155.1 & 30596.5 & 15675.6 & 7.6 & 9.7 \\
\hline 45 & 58 & 2 & 1934.1 & 1256.2 & 1868.7 & 1159.9 & 3.5 & 8.3 \\
\hline 45 & 63 & 4 & 5937.8 & 4121.3 & 5457.6 & 3739.8 & 8.8 & 10.2 \\
\hline \multicolumn{7}{|c|}{ Mean value of error } & 7.3 & 9.1 \\
\hline
\end{tabular}

$$
\text { Note: Relative dviation } \frac{\mid \text { Relative deviation - experimental data| }}{\text { experimental data }} \cdot 100 \%
$$

The range analysis method is used to analyze the test results, the results are shown in Table 6 , the order of significant influence on normal intrusive force and tangential cutting force is obtained as follows: Cutting thickness $\mathrm{h}>$ half cone angle of alloy head beta $>$ cutting angle $\alpha$, this is consistent with the theoretical results, at the same time, the theoretical model of tangential cutting force and normal intrusion force has errors of $9.1 \%$ and $7.8 \%$ from the experimental data, which can meet the engineering application requirements.

Table6 Range analysis of orthogonal test results

\begin{tabular}{|c|c|c|c|c|c|c|}
\hline \multirow{2}{*}{ level } & \multicolumn{2}{|c|}{$\beta 1^{\circ}$} & \multicolumn{2}{|c|}{$\alpha /{ }^{\circ}$} & \multicolumn{2}{|c|}{$h / \mathbf{m m}$} \\
\hline & $F_{n} / \mathbf{k N}$ & $F_{c} / \mathbf{k N}$ & $F_{n} / \mathbf{k N}$ & $F_{c} / \mathbf{k N}$ & $F_{n} / \mathbf{k N}$ & $F_{c} / \mathbf{k N}$ \\
\hline 1 & 2570 & 2436.5 & 11701 & 6736.6 & 1196 & 823.3 \\
\hline 2 & 5085 & 3843.9 & 4203 & 3437.5 & 4804 & 3276.3 \\
\hline 3 & 12048 & 6858.4 & 3799 & 2964.7 & 13703 & 9039.3 \\
\hline range & 9478 & 4421.9 & 7902 & 3771.9 & 12507 & 8216 \\
\hline patch & 2 & 2 & 3 & 3 & 1 & 1 \\
\hline
\end{tabular}




\subsection{Conclusion}

(1) Based on the rock Coulomb-Mohr failure criterion, through theoretical analysis, rock static pressure and shear test, and considering the friction between pick and rock, the cutting force prediction model of pick-type pick with rock failure parameters, geometric parameters of pick and installation parameters as variables is established.

(2) Through cutting test data, the velocity compensation factor of cutting force model is calculated. The maximum error between the calculated cutting process curve and the test curve is about $10 \%$, which has high consistency and consistency, and meets the needs of Engineering data. Significance of Cutting Force: Cutting Thickness > Semi-cone Angle Beta > Cutting Angle alpha.

\section{References}

1. C. Balci, N. Bilgin, Correlative study of linear small and full-scale rock cutting tests to select mechanized excavation machines $[\mathrm{J}]$.International Journal of rock Mechanics and Mining Science, 2007,44 (3):468-476

2. N. Bilgin, M. A. Demircin, H. Copur, C. Balci, H. Tuncdemir, N. Akcin, Dominant rock properties affecting the performance of conical picks and the comparison of some experimental and theoretical results[J]. International Journal of rock Mechanics \& Mining Sciences, 2006, 43(1):139-156

3. Evans, A theory of the cutting force for point attack picks [J]. International Journal of Mining Engineering, 1984,2(1):63-71

4. R.M. Goktan, A suggested improvement on Evans' cutting theory for conical bits [A]. .Brisbane, Queensland, 1997: 57-61

5. R.M. Goktan, N. Gunes, Semi-empirical approach to cutting force prediction for Point-attack picks [J].
Journal of the South African Institute of Mining and Metallurgy, 2005, 105(4):257-263

6. R.H. Bao, L.C. Zhang, Q.Y. Yao, Estimating the peak indentation force of the edge chipping of rocks using single point attack pick [J]. Rock Mechanics and Rock Engineering, 2011, 44(3): 339-347

7. X. Wang, Y. P. Liang, Q. F. Wang, Empirical models for tool forces prediction of drag-typed picks based on principal component regression and ridge regression methods [J].Tunnelling and Underground Space Technology,2017,62: 75-95

8. Y.P. Liang, X. Wang, Q.F Wang, Effects of cut depth and cut spacing on tool forces acting on a conical pick in rock cutting [J]. Journal of vibration and shock, 2018, 3(37):27-33.

9. L. P. Wang, B. S. Jiang, Y. Zhang, Calculation of peak cutting force of conical picks based on Evans' cutting model [J].Journal of China Coal Society,2016,41( 9):2367-2372

10. L. P. Wang, B. S. Jiang, Y. Zhang, Calculation of peak cutting force of conical picks under conditions of dissymmetrical slotting[J]. Journal of China Coal Society,2016,41(11):2876-2882

11. CH. S. Liu, D. G. Li, Mathematical model of cutting force based on experimental conditions of single pick cutting Journal of China Coal Society, 2011,9(36):1565-1569

12. F. L. Tang, Core Drilling[M].Wuhan: China University of Geosciences,1997

13. X. H. XU, J. YU, Rock Crushing[M]. Beijing: China Coal Industry Publishing House, 1984

14. J. X. Liu, CH. Ma, Q.L. Zeng. Research on cutting force of conical pick in cutting coal process [J].Journal of China Coal Society, 2017, 42(5):13251330 